\title{
Correlation between incremental remifentanil doses and the Nociception Level (NOL) index response after intraoperative noxious stimuli
}

\section{Corrélation entre des doses croissantes de rémifentanil et la réponse de l'indice de nociception NOL après des stimuli peropératoires douloureux}

\author{
Etienne Renaud-Roy, MD • Pierre-André Stöckle, MD, MSc, DESAR - Sarah Maximos, MSc, MD • \\ Véronique Brulotte, MD, MSc, FRCPC · Lucas Sideris, MD, FRCSC · Pierre Dubé, MD, MSc, FRCSC, \\ FACS $\cdot$ Pierre Drolet, MD, FRCPC $\cdot$ Issam Tanoubi, MD, MA(ed), DESAR $\cdot$ Rami Issa, BEng, MD, \\ FRCPC • Olivier Verdonck, MD, MSc, DESAR • Louis-Philippe Fortier, MD, MSc, FRCPC • \\ Philippe Richebé, MD, PhD, DESAR
}

Received: 2 November 2018/Revised: 16 February 2019/Accepted: 18 February 2019/Published online: 17 April 2019

(C) Canadian Anesthesiologists' Society 2019

\begin{abstract}
Background The Pain Monitoring Device (PMD) monitor (Medasense Biometrics Ltd., Ramat Gan, Israel) uses the Nociception Level (NOL) index, a multiple parameterderived index that has recently shown a good sensitivity and specificity to detect noxious stimuli. The aim of this study was to assess the latest version of the device $\left(P M D 200^{T M}\right)$ on variations of the NOL response after standardized tetanic stimuli to study the correlation between remifentanil doses and NOL.
\end{abstract}

Electronic supplementary material The online version of this article (https://doi.org/10.1007/s12630-019-01372-1) contains supplementary material, which is available to authorized users.

E. Renaud-Roy, MD - P.-A. Stöckle, MD, MSc, DESAR S. Maximos, MSc, MD - V. Brulotte, MD, MSc, FRCPC P. Drolet, MD, FRCPC · I. Tanoubi, MD, MA(ed), DESAR · R. Issa, BEng, MD, FRCPC · O. Verdonck, MD, MSc, DESAR - L.-P. Fortier, MD, MSc, FRCPC · P. Richebé, MD, PhD, DESAR $(\square)$

Department of Anesthesiology of Maisonneuve-Rosemont Hospital, CIUSSS de l'Est de l'Ile de Montreal, University of Montreal, 5415 Boulevard de l'Assomption, Montreal, QC H1T 2M4, Canada

e-mail: philippe.richebe@umontreal.ca

L. Sideris, MD, FRCSC · P. Dubé, MD, MSc, FRCSC, FACS Department of Surgery, Maisonneuve-Rosemont Hospital, CIUSSS de l'Est de l'Ile de Montreal, University of Montreal, Montreal, QC, Canada
Methods Data from 26 patients undergoing midline laparotomy and receiving a desflurane-remifentanil-based anesthetic coupled with low thoracic epidural analgesia were analyzed. A standardized tetanic stimulus was applied to the forearm of the patients at different remifentanil infusion rates. The primary aim was to evaluate the correlation between post-tetanic stimulation NOL values from the PMD200 and remifentanil doses. The NOL index variations after experimental and clinical stimuli were also compared with heart rate (HR), mean arterial pressure (MAP), and Bispectral Index ${ }^{T M}(B I S)$.

Results A correlation between post-tetanic stimulation NOL values and remifentanil doses was found $(r=-0.56$; 95\% confidence interval [CI], -0.70 to $-0.44 ; P<0.001)$. The NOL discriminated noxious from non-noxious states with the maximal Youden's index value of the NOL receiver operating characteristic (ROC) curve showing a specificity of $88 \%$ (95\% CI, 69.0 to 100) and sensitivity of $79.1 \%$ (95\% CI, 56.2 to 95.5). The area under the NOL ROC curve (AUC, 0.9; 95\% CI, 0.84 to 0.95) was significantly different from the other variables $(P<0.001$ vs HR; $P<$ 0.001 vs MAP; $P<0.001$ vs BIS).

Conclusions The NOL value after noxious stimulus decreased with incremental remifentanil doses, showing a significant inverse correlation between the NOL index and opioid doses. The sensitivity and specificity of NOL to discriminate between noxious and non-noxious stimuli suggests its interesting potential as a monitor of nociception intensity during anesthesia. 
Trial registration $w w w$.clinicaltrials.gov

$(N C T$

02884778); 27 July, 2016.

\section{Résumé}

Contexte Le moniteur de la douleur PMD (Pain Monitoring Device) (Medasense Biometrics Ltd., Ramat Gan, Israël) se fonde sur l'indice NOL (Nociception Level), un indice dérivé de paramètres multiples dont la sensibilité et la spécificité se sont avérées élevées pour détecter les stimuli douloureux. L'objectif de cette étude était d'évaluer la dernière version du dispositif (PMD200 $\left.{ }^{T M}\right)$ sur des variations de la réponse NOL après des stimuli tétaniques normalisés afin d'étudier la corrélation entre des doses des rémifentanil et le NOL.

Méthode Les données de 26 patients subissant une laparotomie médiane et recevant une anesthésie de desflurane-rémifentanil ainsi qu'une analgésie par péridurale thoracique basse ont été analysées. Un stimulus tétanique normalisé a été appliqué à l'avant-bras des patients recevant des perfusions de rémifentanil à différentes doses. L'objectif principal était d'évaluer la corrélation entre les valeurs de NOL après la stimulation tétanique relevées par le PMD200 et les doses de rémifentanil. Les variations de l'indice NOL après les stimuli expérimentaux et cliniques ont également été comparées à la fréquence cardiaque (FC), à la tension artérielle moyenne (TAM), et à l'indice bispectral ${ }^{T M}$ (BIS). Résultats Une corrélation a été observée entre les valeurs NOL post stimulation tétanique et les doses de rémifentanil ( $r=-0,56$; intervalle de confiance [IC] $95 \%,-0,70$ à -0,44; $P<0,001)$. Le NOL a distingué les états nociceptifs des états non nociceptifs, la valeur maximale de l'indice de Youden de la courbe ROC (courbe caractéristique efficace - receiver operating characteristic) montrant une spécificité de $88 \%$ (IC 95\%, 69,0 à 100) et une sensibilité de 79,1 \% (IC 95\%, 56,2 à 95,5). La surface sous la courbe ROC de NOL (SSC, 0,9; IC 95\%, 0,84 à $0,95)$ était significativement différente par rapport aux autres variables $(P<0,001$ vs $F C ; P<0,001$ vs TAM; $P<$ 0,001 vs BIS).

Conclusion La valeur NOL après un stimulus nociceptif a baissé avec des doses croissantes de rémifentanil, ce qui démontre une corrélation inverse significative entre l'indice NOL et les doses opiö̈des. La sensibilité et la spécificité du NOL pour distinguer les stimuli nociceptifs et non nociceptifs suggèrent que cet indice pourrait être intéressant pour monitorer l'intensité de la nociception pendant l'anesthésie.

Enregistrement de l'étude $w w w$.clinicaltrials.gov (NCT 02884778); 27 juillet 2016.
Anesthesiologists aim to achieve four essential objectives with general anesthesia (GA): unconsciousness (lack of awareness), amnesia (lack of memory), akinesia (lack of movement), and antinociception. The monitoring of muscle relaxation $^{1}$ and depth of unconsciousness ${ }^{2}$ has been extensively studied and produces relatively reliable and sensitive devices and indexes compared with common clinical signs. Nevertheless, monitoring of antinociception, defined as inhibition of the nociceptive afferent pathways, remains a major intraoperative challenge. Indeed, anesthesiologists usually have to infer the nociceptive level from clinical signs such as hypertension and tachycardia that are linked to the activation of the autonomic nervous system (ANS).

The deleterious acute neuroendocrine response ${ }^{3}$ of ANS activation-with surgical stress inducing increases in cortisol secretion from adrenal cortex, arginine vasopressin, aldosterone, adrenocorticotropic hormone, growth hormone, etc.- is not the only reason for tight control of nociception. Reducing nociceptive inputs may prevent the development of chronic pain syndromes including persistent postsurgical pain. ${ }^{4,5}$ Furthermore, it has been shown that overtreatment with opioid analgesics leads to opioid-induced hyperalgesia, ${ }^{6,7}$ higher opioid tolerance, ${ }^{8}$ and immunosuppression. ${ }^{9-11}$

Despite an understanding of nociception and its related morbidity, the development of an accurate monitoring device for detection of nociception remains challenging. In the past two decades, new parameters have been studied that have mostly evaluated the level of activation of the sympathetic $\mathrm{ANS}^{12}$; skin conductance (SC), electroencephalography, electromyography, photoplethysmographic pulse wave amplitude (PPGA), and heart rate variability (HRV) have all been evaluated. ${ }^{13-19}$ Device algorithms have largely been based on the analysis of a single parameter, and have shown inter-individual variability to be a major limiting factor in their daily use during GA. ${ }^{20,21}$

Some multi-parameter devices are superior to their single-parameter competitors, particularly in correctly discriminating between the mild to moderate pain stimuli. $^{22,23}$ A multi-parameter approach combining HRV, PPGA, state entropy, and response entropy generated an index capable of estimating the nociceptiveanti-nociceptive balance at skin incision during GA. ${ }^{24}$

Ben-Israel et al. published the first study on the Nociception Level (NOL) index in 2013. The NOL index is generated from a non-linear random forest regression using five different parameters gathered from a finger probe connected to a device called the Pain Moderating 
Device (PMD) $100^{\mathrm{TM}}$ (Medasense Biometrics Ltd, Ramat Gan, Israel). ${ }^{25}$ These parameters include heart rate (HR), $\mathrm{HRV}$ at the $0.15-$ to $0.4-\mathrm{Hz}$ band power, PPGA, SC level, number of SC fluctuations, and their time derivatives. The output, a single dimensionless number from 0 to 100 , correlated proportionally to nociception. The NOL index has a high sensitivity and specificity to discriminate noxious stimuli and their intensity under GA. ${ }^{26,27}$ The NOL correlated with clinical responses during intubation and was less reactive if remifentanil was administered. ${ }^{28}$ These studies have also shown a better sensitivity of the NOL index to detect noxious stimulus than changes in HR, mean arterial pressure (MAP), Bispectral Index ${ }^{\mathrm{TM}}$ (BIS), and PPGA. ${ }^{26-28}$ The PMD200 ${ }^{\mathrm{TM}}$ is the latest version of this monitor and is notable for having more efficient sensors and an integrated analog-to-digital converter.

Using this new version PMD200, the primary objective of the present study was to analyze the intra and interindividual variability of NOL response after clinical and experimental noxious stimuli at various incremental doses of intravenous remifentanil infusion. We hypothesized that there would be a negative and significant correlation between NOL values and incremental intravenous remifentanil infusion doses following standardized repetitive noxious stimuli. As secondary endpoints, we also compared the sensitivity and the specificity of the NOL index with individual parameters such as HR, MAP, and BIS.

\section{Methods}

This trial was conducted at the Maisonneuve-Rosemont University Hospital, University of Montreal, Montreal, QC, Canada, between April and July 2016. The study was approved by the local and Scientific and Ethics Committee (Comité d'Ethique de la Recherche, installation Hôpital Maisonneuve-Rosemont, CIUSS de l'Est de l'île de Montréal, Montréal, QC, Canada; CER 15074; 22 March, 2016) and was registered at clinicaltrials.gov (NCT02884778). All patients provided signed informed consent.

Inclusion and exclusion criteria

Adult patients (age $\geq 18 \mathrm{yr}$ ) with American Society of Anesthesiologists physical status I-III, scheduled to undergo elective abdominal surgery with laparotomy under GA and epidural analgesia, were screened. The exclusion criteria included a history of coronary artery disease, serious cardiac arrhythmia (including atrial fibrillation), history of substance abuse, chronic use of psychotropic and/or opioid drugs, use of drugs that act on the ANS (such as $\beta$-blockers), history of psychiatric diseases or psychologic problems, and allergy to any drug used in the study protocol. In addition, patients were also excluded if an unexpected difficult airway was encountered requiring painful airway manipulations, dural puncture during epidural catheter installation, or if unexpected intraoperative patient instability requiring significant hemodynamic support (transfusions, vasopressors, inotropes) occurred.

\section{Procedures}

\section{EPIDURAL PLACEMENT}

The timeline of the various study procedures is described in Fig. 1. Intravenous $0.02 \mathrm{mg} \mathrm{kg}^{-1}$ midazolam (Fresenius Kabi, Toronto, ON, Canada) was administered prior to the epidural catheter placement. The epidural catheter was inserted with the patient in the sitting position in the operating room (OR) before GA. This catheter was first tested with aspiration using a 1-mL syringe, and then a 3$\mathrm{mL}$ bolus of saline was injected to test its patency. At this time, no local anesthetic was injected, which allowed the first experimental stimulus in the study protocol to be performed prior to any epidural loading.

\section{Induction of GA}

The patient was then placed in a supine position and a PMD200 monitor and BIS ${ }^{\circledR}$ (Medtronic, Kirkland, QC, Canada) were applied and connected for continuous recording. General anesthesia was then induced with 1.5-2 $\mathrm{mg} \cdot \mathrm{kg}^{-1}$ propofol (Pharmascience Inc, Montreal, QC, Canada), remifentanil (Teva, Stouffville, Canada) bolus (1 $\left.\mu \mathrm{g} . \mathrm{kg}^{-1}\right)$, followed by a continuous infusion of 0.05 $\mu \mathrm{g} \cdot \mathrm{kg}^{-1} \cdot \mathrm{min}^{-1}$ and $1 \mathrm{mg} \cdot \mathrm{kg}^{-1}$ rocuronium (Sandoz Canada Inc, Boucherville, QC, Canada). Intubation was performed three minutes after the beginning of the treatment with propofol, remifentanil, and rocuronium boluses. All drugs were given according to the adjusted patient's body weight $(\mathrm{ABW})$, which was calculated with the Robinson's formula: $\quad A B W=I B W+[0.4 \times(T B W-I B W)]$ where $T B W$ is total body weight and $I B W$ is ideal body weight. ${ }^{29-31}$

Immediately after intubation, anesthesia was maintained with desflurane, adjusting the end-expiratory fraction to maintain a BIS value between 40 and 60 . Three minutes after this first clinical stimulus (i.e., intubation), the remifentanil infusion was decreased to 0.005 $\mu \mathrm{g} \cdot \mathrm{kg}^{-1} \cdot \mathrm{min}^{-1}$ to reduce the risk of developing hypotensive events and to prepare the patient for the first experimental stimulus of the study protocol.

A radial artery catheter was then inserted to continuously monitor the patient's arterial blood pressure 


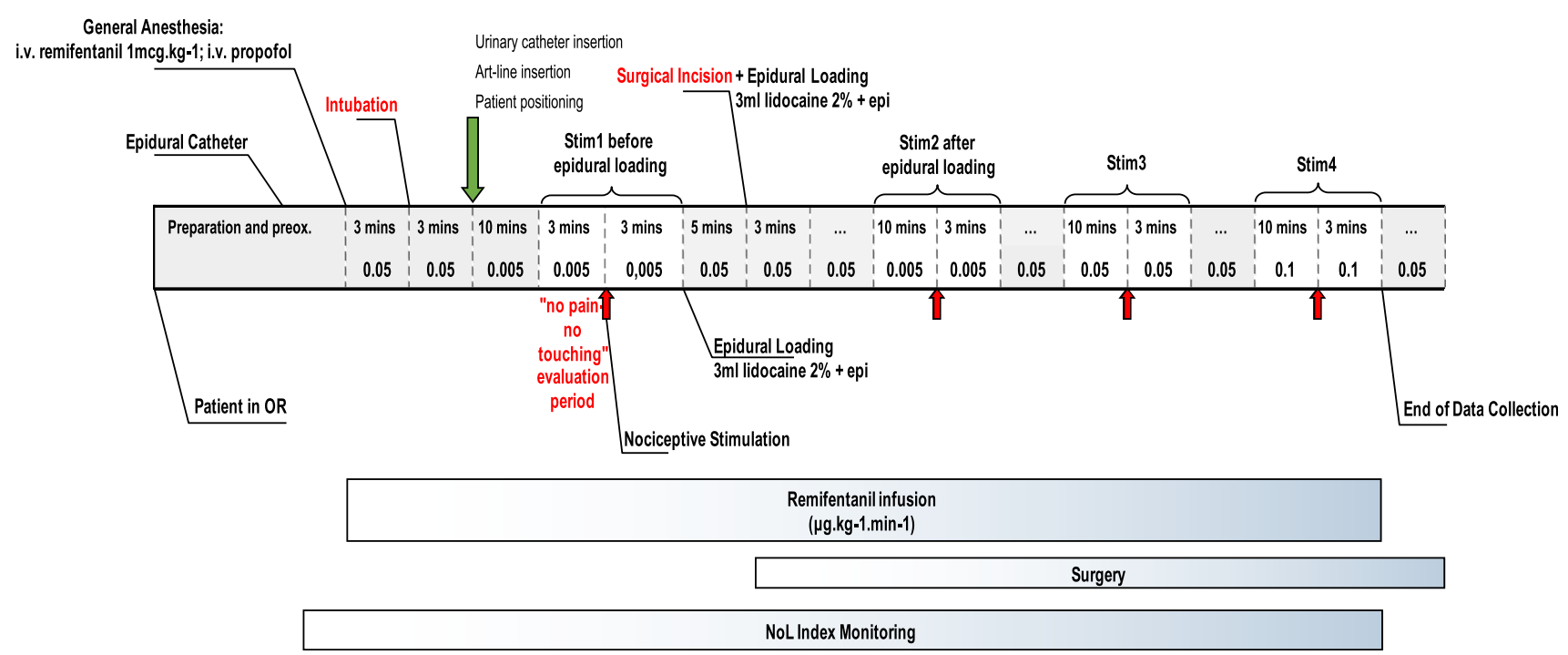

Fig. 1 Data collection timeline showing all the steps for general anesthesia induction and maintenance. Clinical stimuli, such as intubation and incision, are represented. Green arrow between intubation and incision represents the patient preparation (urinary catheter, arterial line placement, etc.). "No pain-no touching" evaluation period was the time during which baseline blood

and calculate delta pulse pressure to infer intravascular volume status (as all enrolled patients were in the enhanced recovery after surgery program in our institution).

\section{Experimental stimuli}

At least ten minutes after the post-intubation decrease in the remifentanil infusion, and once the arterial line was inserted, the OR team stopped any physical contact/ stimulation of the patient allowing at least a three-minute rest period under stable anesthesia. Baseline MAP and HR were defined for the rest of the study as the mean of three measures in the three minutes during this "no pain-no touching" evaluation period (Fig. 1) to get baselines values of all the study parameters. If necessary, phenylephrine was given to keep a MAP $>65 \mathrm{mmHg}$.

At this point, the first standardized electrical (tetanic) stimulation was applied on the area of the ulnar nerve at the wrist level, at a frequency of $100 \mathrm{~Hz}$ and a current of $70 \mathrm{~mA}$ for a duration of $30 \mathrm{sec}$, followed by a three-minute observation period (EZstimII, Model ES400, Life-Tech, Stafford, TX, USA). This type of noxious stimulation was used in other studies without any reported side effects. ${ }^{32}$ Once the observation period ended after this first electrical stimulus (after three minutes), the epidural catheter was bolused with 3 $\mathrm{mL}$ of lidocaine $2 \%$ with epinephrine (AstraZeneca Inc, Mississauga, ON, Canada) in preparation for the incision. Approximately five minutes after this first epidural dose, and under a continuous remifentanil infusion at 0.05 $\mu \mathrm{g} \mathrm{kg}^{-1} \mathrm{~min}^{-1}$, the incision for laparotomy was performed, pressure and heart rate values were recorded. Small red arrows on the bottom part of the timeline represent the experimental nonoperator dependent stimulations called Stim1, 2, 3, and 4 in the text. Stim1 was always performed before epidural first bolus; Stim2, 3, and 4 were done after epidural was bolused (Color figure online)

and all data were recorded electronically for a three-minute period. Immediately before the abdominal incision, a second 3mL epidural bolus of lidocaine $2 \%$ was given. After incision, an increase of more than $20 \%$ in HR and/or MAP was considered as inadequate epidural analgesia. In this case, an additional 3$\mathrm{mL}$ epidural bolus of lidocaine $2 \%$ solution could be given to achieve adequate analgesia for surgery.

During the surgery, both MAP and HR increases were also used to guide the administration of extra intravenous boluses of remifentanil by the attending anesthesiologist. If mean MAP or HR increased to over $20 \%$ of their baseline (pre-incision) values, a $0.3 \mu \mathrm{g} \cdot \mathrm{kg}^{-1}$ remifentanil bolus was administered.

Once stable hemodynamic conditions were achieved for a ten-minute period without additional remifentanil bolus, a second, third, and fourth tetanic standardized and identical electrical stimulation was performed at a remifentanil dosage of $0.005,0.05$, and $0.1 \mu \mathrm{g} \mathrm{kg}^{-1} \mathrm{~min}^{-1}$, respectively. For each electrical stimulus, the remifentanil infusion rate was kept the same for at least ten minutes prior to the stimulation to make sure that the plasma concentration was steady. During the sixminute period surrounding each electrical stimulus (three minutes before and three minutes after), any patient contact by the OR, anesthesia, and surgical team was temporarily interrupted. Surgery resumed three minutes after the stimulus application.

\section{Data collection}

All the intraoperative data were recorded with an automated information management system. The 
PMD200 monitor device recorded intraoperative NOL values every five seconds. Other intraoperative parameters (HR, MAP, BIS, $\mathrm{SpO}_{2}$, temperature, etc.) as well as respiratory parameters and volatile anesthetic concentrations and consumption (in $\mathrm{mL}$ ) were automatically and electronically collected every second from the Perseus ${ }^{\circledR}$ A500 anesthesia workstation connected to the physiologic monitor M540 (Dräger AG \& Co., Lübeck, Germany). At the end of the procedure, all data were de-identified, transferred, and stored into a computer data file for further statistical analysis.

\section{Statistical analysis}

We expected that increasing analgesia with the infusion of remifentanil would negatively correlate to NOL index variation after experimental electrical stimulations. To reach a Spearman's correlation $r$ value of -0.5 (based on previously published studies), ${ }^{33,34}$ with a power of $1-\beta=$ $80 \%$, and a type I error of $\alpha=0.05$, we determined that 29 patients would be needed to reach our primary objective. We decided to include 30 patients.

We also hypothesized that the response of all parameters to tetanic stimulus would decrease when increasing remifentanil infusion rate and we explored the NOL index ability to detect tetanic stimulation under higher remifentanil infusion dosage. For intubation and incision, as well as for each tetanic stimulation, parameters values (NOL, HR, MAP, and BIS) for the three minutes before stimulation as well as the three minutes following the stimulation were recorded. All pre-stimulation parameter data were defined as the average of the 30 -sec period right before a clinical or experimental noxious stimulation. The post-stimulation parameters values were defined as the average of the 10-80 sec data points (which includes the peak for each parameter) after the noxious stimulation.

Ordinal and continuous data were tested for normality using Shapiro-Wilk test, and if probably non-normal (considering an alpha $=0.05$ ), were presented as medians [interquartile range]. Categorical data were presented as counts and percentages. Normal data were presented as mean (standard deviation [SD]).

The Spearman correlation coefficient was calculated to assess whether pre-stimulation and post-stimulation measures of experimental stimulus were respectively correlated with increasing remifentanil infusion rate.

Post-stimulation responses to clinical (i.e., intubation and incision) and experimental stimuli were compared with their respective pre-stimulation measures using Wilcoxon rank signed test with Bonferroni correction for six comparisons (two clinical and four experimental stimulations) for each of the studied parameters. The responses to the experimental stimuli during incremental remifentanil infusion rate were compared using the Friedman's test. A post hoc analysis using DunnBonferroni test was carried out to determine significant differences within the increment of remifentanil infusion.

Receiver operating characteristic (ROC) curves were constructed to evaluate the ability of the individual parameter to discriminate between noxious and nonnoxious states and confidence intervals of the area under the curves (AUCs) were calculated. Non-parametric bootstrap analysis was performed on the NOL values and was repeated 1,000 times. For each set of 1,000 bootstrap replicates, we obtained the optimal sensitivity, specificity, and their corresponding cut-off value, according to the maximal Youden's index. As for the 1,000 values of sensitivity, specificity, and cut-offs, we obtained a single confidence interval. For HR, MAP, and BIS index values, we used the optimal NOL specificity as the threshold at each of the 1,000 bootstrap iterations.

To assess the evolution of the NOL response to experimental noxious stimulation at different remifentanil infusion rates, the patients' average values for each fivesecond recording period were plotted against time. Positive AUCs were analyzed using the Friedman test. Post hoc analysis using the Dunn-Bonferroni test was performed to assess significant differences in AUCs at the different remifentanil infusion dosages.

All statistical analyses were performed using IBM SPSS Statistics for Mac version 24.0 (IBM Corp., Armonk, NY, USA).

\section{Results}

Study population

Among the 32 patients screened, 30 were recruited and data from 26 were included for full analysis. Four patients were excluded from data analyses because of incomplete data. One patient developed intraoperative bigeminy short after induction of anesthesia and was excluded because the HR and NOL were no longer stable. One patient had an intravascular epidural catheter that was discovered at the time of incision so was excluded. Two patients had very poor quality NOL signals (due to cold fingers) and were also excluded from analyses (Fig. 2).

The patient mean (SD) age was 59 (13) yr and almost two-thirds were female (Table 1). Most (81\%) participants underwent gastrointestinal abdominal surgical procedures with one-third undergoing midline laparotomies for tumor resection and hyperthermic intraperitoneal chemotherapy. Other participants underwent gynecologic and urologic procedures with abdominal midline vertical incision. 


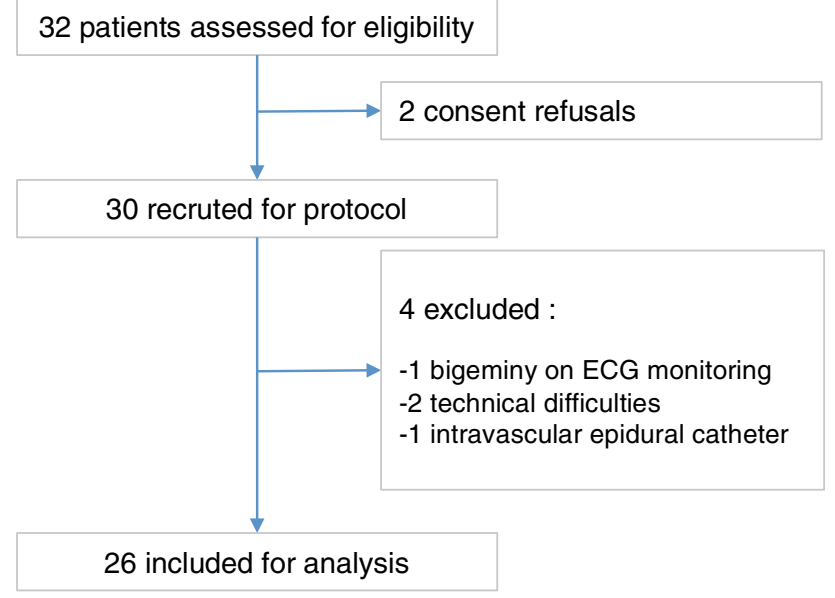

Fig. 2 Flow-chart of the study

Table 1 Characteristics of study population

\begin{tabular}{ll}
\hline & Total $(n=26)$ \\
\hline Age $(\mathrm{yr})$ & $59(13)$ \\
Sex M/F & $9 / 17$ \\
Height $(\mathrm{cm})$ & $166(9)$ \\
Weight $(\mathrm{kg})$ & $69.1(12.3)$ \\
BMI $\left(\mathrm{kg} \cdot \mathrm{cm}^{-2}\right)$ & $25.2(4.5)$ \\
ASA I & $3(12 \%)$ \\
ASA II & $21(81 \%)$ \\
ASA III & $2(8 \%)$ \\
Type of laparotomy & \\
HIPEC & $9(35 \%)$ \\
Other general & $12(46 \%)$ \\
Urology & $2(8 \%)$ \\
Gynecology & $3(12 \%)$
\end{tabular}

Values presented as mean (SD). ASA and type of surgery presented as $n(\%)$.

ASA $=$ American Society of Anesthesiologists; BMI = Body mass index; HIPEC = hyperthermic intraperitoneal chemotherapy.

Nociception and antinociception

Nociception Level index values after experimental tetanic electrical stimulation were negatively correlated with incremental remifentanil infusion dose $(\mathrm{r}=-0.56 ; 95 \%$ confidence interval $[\mathrm{CI}],-0.70$ to $-0.44 ; P<0.001)$. The negative correlation between remifentanil infusion dose and post-stimulation $\mathrm{HR}(\mathrm{r}=-0.42 ; 95 \% \mathrm{CI},-0.57$ to $-0.24 ; P<0.001)$ and MAP $(\mathrm{r}=-0.32 ; 95 \% \mathrm{CI},-0.47$ to $-0.10 ; P=0.002)$ were significant for both. There was no significant correlation between the post-stimulation BIS values and the doses of remifentanil infusion $(r=-0.03$; 95\% CI, -0.29 to $0.10 ; P=0.77$ ) (eAppendix 1 , available as Electronic Supplementary Material [ESM]).
Results of pre- and post-stimulation values for all parameters are shown in Table 2 and Fig. 3 (also see eAppendices 1 and 2 available as ESM). The NOL values significantly increased following the clinical stimuli, intubation and incision, reaching 17 and 7, respectively.

The HR values significantly increased after intubation (performed at the remifentanil infusion dose of 0.05 $\mu \mathrm{g} \mathrm{kg}^{-1} \mathrm{~min}^{-1}$ and three minutes after induction drugs were administered) and after experimental stimulation at the lowest remifentanil infusion dose $0.005 \mu \mathrm{g} . \mathrm{kg}^{-1} \cdot \mathrm{min}^{-1}$ (paired analyses) but neither after the incision (at remifentanil infusion dose $0.05 \mu \mathrm{g} \cdot \mathrm{kg}^{-1} \cdot \mathrm{min}^{-1}$ ) nor after tetanic stimulation with the higher doses of remifentanil (0.05 or $0.1 \mu \mathrm{g} \cdot \mathrm{kg}^{-1} \mathrm{~min}^{-1}$ ).

As there was no radial arterial catheter placed before anesthesia induction, there was no measurement linking MAP to intubation since non-invasive blood pressure was measured each minute, which is not a frequent enough interval for data analysis. Nevertheless, MAP data were available after intubation and for the rest of the study, recorded every five seconds. The MAP did not significantly increase after incision or after tetanic stimulation with the two highest doses of remifentanil $(0.05$ or 0.1 $\left.\mu \mathrm{g} \cdot \mathrm{kg}^{-1} \cdot \min ^{-1}\right)$. The BIS did not change after the clinical and experimental stimulations.

Fig. 4A presents trends of NOL values during the recording period of each experimental stimulation and their AUC (Fig. 4B, and eAppendix 3 in the ESM).

The NOL responses to clinical stimuli such as intubation and incision are shown in Fig. 5. The AUC of the NOL index response significantly decreased when higher doses of intravenous remifentanil infusion were administered (Fig. 5, and eAppendices 3, 4, and 5 in the ESM). The maximal NOL values for experimental stimulation were observed 40-90 sec after the stimuli before recovering to their pre-stimulation baseline values. The correlation between AUC of NOL measures after experimental stimuli at different remifentanil dosages was significantly negative ( $r=-0.60 ; 95 \% \mathrm{CI},-0.71$ to $-0.46 ; P<0.001$ ).

The NOL and individual parameters (HR, MAP, and BIS) threshold points at which the optimal sensitivity and specificity were identified (the best operational point) are represented by the ROC plots and show that at low remifentanil concentration $\quad\left(0.005 \mu \mathrm{g} \cdot \mathrm{kg}^{-1} \mathrm{~min}^{-1}\right.$, following epidural), the NOL index's sensitivity was better than HR, MAP, and BIS (tetanic stimulation \#2; Fig. 2). The maximal Youden's index value of the NOL ROC curve was calculated and reached a specificity of $88 \%$ (95\% CI, 69.0 to 100$)$ and a sensitivity of $79.1 \%$ (95\% CI, 56.2 to 95.5 ), corresponding to a cut-off median value of 10 (95\% CI, 5.3 to 16.3). The sensitivity for HR, MAP, and BIS was $19.1 \%$ (95\% CI, 3.7 to 43.3 ), $35 \%$ (95\% CI, 12.2 to 54.3 ), and $15.5 \%$ (95\% CI, 1.9 to 30.4 ), respectively, and 
Table 2 Pre/post stimulus measures and trends at clinical and experimental stimuli

\begin{tabular}{|c|c|c|c|c|c|c|}
\hline & Intubation & Incision & Stim1 & Stim2 & Stim3 & Stim4 \\
\hline $\begin{array}{l}\text { Remifentanil dosage } \\
\qquad\left(\mu \mathrm{g} \cdot \mathrm{kg}^{-1} \cdot \mathrm{min}^{-1}\right)\end{array}$ & bolus $1 \mu \mathrm{g} \cdot \mathrm{kg}^{-1}$ then 0.05 & 0.05 & 0.005 & 0.005 & 0.05 & 0.1 \\
\hline Bolused epidural: & No & Yes & No & Yes & Yes & Yes \\
\hline \multicolumn{7}{|l|}{ NOL } \\
\hline$n$ & 25 & 25 & 25 & 25 & 26 & 26 \\
\hline Pre & $5.1[2.9-10.8]$ & $3.3[2.6-5.9]$ & $6.9[2.9-10.5]$ & $3.0[2.0-3.8]$ & $7.6[4.9-13.3]$ & $4.9[3.0-6.1]$ \\
\hline Post & $16.8[7.5-21.4]$ & $6.8[3.9-10.1]$ & 18.6 [14.2-26.9] & $13.3[7.6-25.5]$ & $10.4[5.3-15.9]$ & $5.5[3.0-7.6]$ \\
\hline$P$ value & $0.00027 *$ & 0.0045 & $<0.0001 *$ & $<0.0001 *$ & 0.19 & 0.21 \\
\hline \multicolumn{7}{|l|}{ HR } \\
\hline$n$ & 25 & 25 & 24 & 25 & 25 & 25 \\
\hline Pre & $62.4[55-66]$ & $54.3[47-64]$ & $67.4[58-81]$ & $59.1[52-67]$ & $62.1[51-73]$ & $58.4[48-70]$ \\
\hline Post & $65.7[58-82]$ & $55.5[49-62]$ & $72.8[65-87]$ & $67.3[56-79]$ & $63.5[56-74]$ & $55.3[48-68]$ \\
\hline$P$ value & $0.00045^{*}$ & 0.54 & $<0.0001 *$ & $<0.0001 *$ & 0.59 & 0.054 \\
\hline \multicolumn{7}{|l|}{ MAP } \\
\hline$n$ & & 23 & 23 & 24 & 24 & 24 \\
\hline Pre & & 81.0 [70-89] & 73.6 [66-89] & 77.2 [67-82] & 73.9 [60-81] & 75.2 [69-82] \\
\hline Post & & 83.9 [72-89] & 78.7 [74-92] & 85.7 [78-94] & 74.6 [62-85] & $74.0[69-81]$ \\
\hline$P$ value & & 0.90 & $<0.0001 *$ & $<0.0001 *$ & 0.91 & 0.86 \\
\hline \multicolumn{7}{|l|}{ BIS } \\
\hline$n$ & 23 & 25 & 24 & 24 & 25 & 25 \\
\hline Pre & 30.4 [26-41] & $42.9[40-47]$ & 42.5 [39-50] & $43.1[40-44]$ & 44.1 [41-49] & $43.0[39-45]$ \\
\hline Post & 29.6 [25-36] & $43.1[40-49]$ & $45.5[39-48]$ & $43.2[40-48]$ & 46.3 [41-49] & $43.8[41-45]$ \\
\hline$P$ value & 0.52 & 0.34 & 0.43 & 0.13 & 0.18 & 0.39 \\
\hline
\end{tabular}

Values presented as median [interquartile range]; $\mathrm{BIS}=$ bispectral index; $\mathrm{HR}=$ heart rate; MAP $=$ mean arterial blood pressure; NOL $=$ Nociceptive Level index; Stim $=$ stimulation. $n=$ number of patients analyzed; Stim1, 2, 3, and 4 are represented in Fig. 1 study timeline; $P$ values represent Wilcoxon signed rank test significant at $* P<0.0042$ (paired test; after Bonferroni correction for multiple comparisons of stimuli)

was calculated at an $88 \%$ specificity, according to the maximal Youden index. The area under the NOL ROC curve (AUC, 0.9; 95\% CI, 0.84 to 0.95) exhibited a significant (following Bonferroni correction, three comparison; alpha $=0.0167)$ difference compared with all other variables $(P<0.001$, NOL $v s$ HR; $P<0.001 \mathrm{NOL}$ vs MAP; $P<0.001$ NOL $v s$ BIS) (Table 3, Fig. 6).

\section{Discussion}

In this study, we analyzed the intra- and inter-individual variations of the NOL index response to noxious stimuli under various intravenous remifentanil infusion rates. The main result of our study is the significant inverse correlation between remifentanil infusion rate at the time of the stimulation and the post-stimulation NOL values. At the highest doses of remifentanil, NOL did not change after the tetanic stimulation.

Our study also reports that the NOL index response after a standardized electrical noxious stimulus does not change significantly with the loading of a low thoracic epidural catheter with a dose of $3 \mathrm{~mL}$ of lidocaine $2 \%$ associated with epinephrine.

The significant negative correlation between remifentanil infusion dose and post-stimulation NOL index $(\mathrm{r}=-0.56 ; 95 \% \mathrm{CI},-0.70$ to $-0.44 ; P<0.001)$ also existed for $\mathrm{HR}(\mathrm{r}=-0.42 ; 95 \% \mathrm{CI},-0.57$ to $-0.24, P$ $<0.001)$ and MAP ( $\mathrm{r}=-0.32 ; 95 \% \mathrm{CI},-0.47$ to $-0.10, P$ $=0.002)$. It has to be noted that, at higher remifentanil dosage, the response was not significant with poststimulation NOL index values staying under 10 . In a paired analysis, HR and MAP discriminated experimental tetanic stimuli at the lowest dose of remifentanil and poststimulation values were correlated to remifentanil infusion rate. But in an unpaired test, these parameters were unable to discriminate experimental tetanic stimuli while the NOL index did. At higher remifentanil dosage, neither HR and MAP nor NOL were able to discriminate experimental tetanic stimuli. Bispectral index was unable to discriminate the noxious clinical or experimental stimuli. Moreover, post-stimulation BIS values were not correlated to remifentanil dosage $(\mathrm{r}=-0.03 ; 95 \% \mathrm{CI},-0.29$ to 0.10 ; $P=0.77)$. 

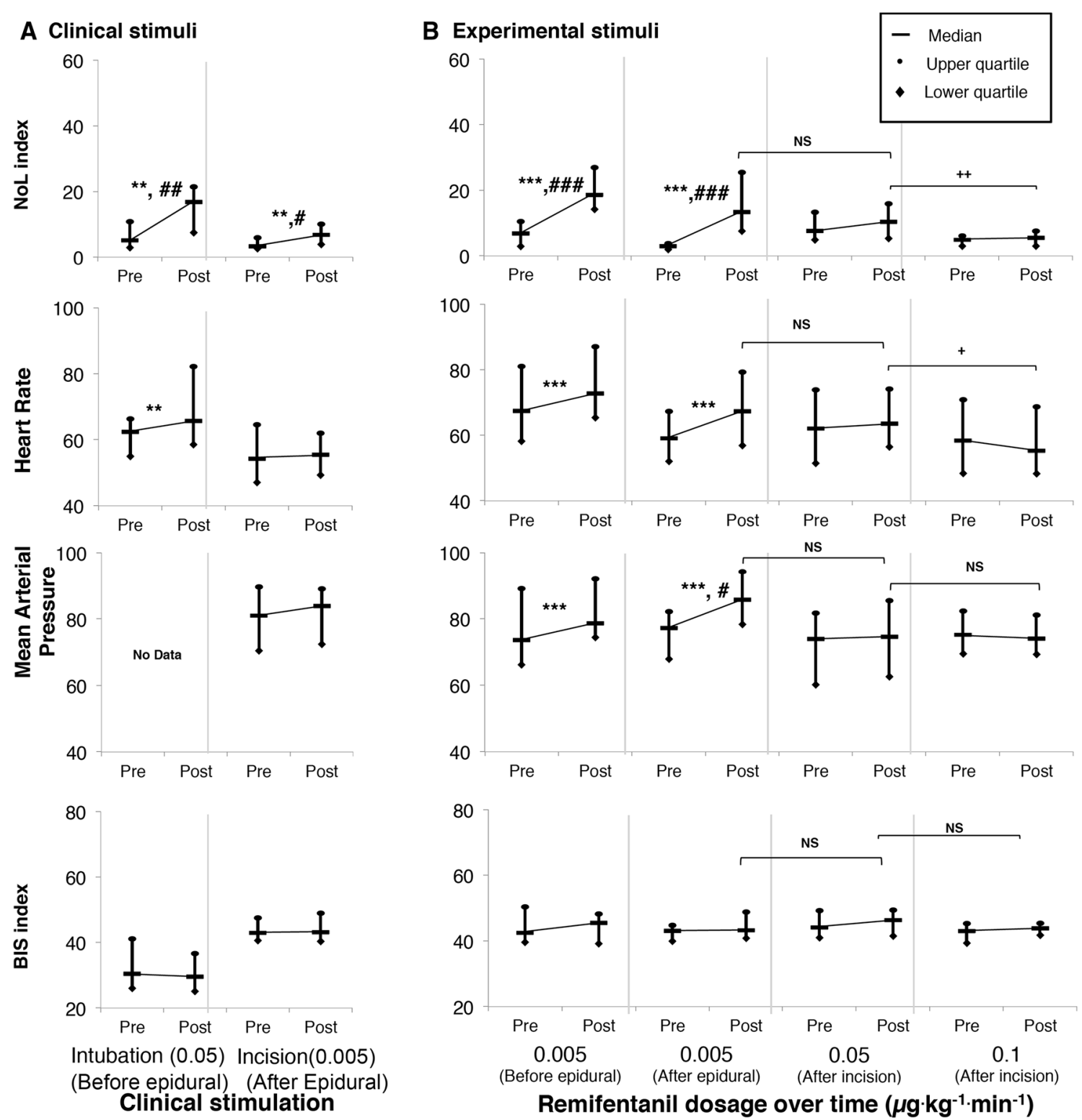

Remifentanil dosage over time $\left(\mu \mathrm{g} \cdot \mathrm{kg}^{-1} \cdot \mathrm{min}^{-1}\right)$

Fig. 3 Pre- to post-stimulation comparison of NOL index, heart rate, mean arterial pressure, and BIS index. Comparison with Wilcoxon rank signed test. $* P<0.0042, * * P<0.001, * * * P<0.0001$ (paired test). Comparison with Mann-Whitney U test (rank sum test). \# $P<$ 0.0125 (Bonferroni correction: four tests), \#\# $P<0.001$, \#\#\# $P<$

The NOL successfully identified the anticipated clinical noxious stimuli (intubation and incision). Even if intubation was performed under rather high remifentanil doses (intravenous continuous infusion set at 0.05 $\mu \mathrm{g} \cdot \mathrm{kg}^{-1} \cdot \mathrm{min}^{-1}$ and three minutes after $1 \mu \mathrm{g} \cdot \mathrm{kg}^{-1}$ remifentanil bolus), the NOL significantly increased after this stimulus, where applying experimental tetanic stimulus at the same dose showed a non-significant response, most likely because experimental tetanic is a milder stimulation. Similarly, the incision was correctly identified as a noxious stimulus by the NOL index, even
0.0001 (unpaired test). Post-hoc analysis with Wilcoxon rank signed test $+P<0.025,++P<0.001 . \mathrm{NOL}=$ Nociception Level Pre $=$ pre-stimulation; Post $=$ post-stimulation. Remifentanil doses are in $\mu \mathrm{g} \cdot \mathrm{kg}^{-1} \mathrm{~min}^{-1}$

following epidural loading. Interestingly, NOL presented a different trend during incision with a more gradual increase and a later peak compared with the other stimulations.

Nociception Level index ROC curve AUC values (AUC, $0.90 ; 95 \%$ CI, 0.84 to 0.95 ) for discrimination between noxious and non-noxious and built from data gathered at remifentanil infusion of $0.005 \mu \mathrm{g} \cdot \mathrm{kg}^{-1} \cdot \mathrm{min}^{-1}$ after epidural loading (stimulation 2, see Fig. 2) were consistent with previous studies and were better than HR (AUC, 0.65; 95\% CI, 0.55 to 0.75 ), MAP (AUC, $0.68 ; 95 \%$ CI, 0.59 to 0.78 ), 


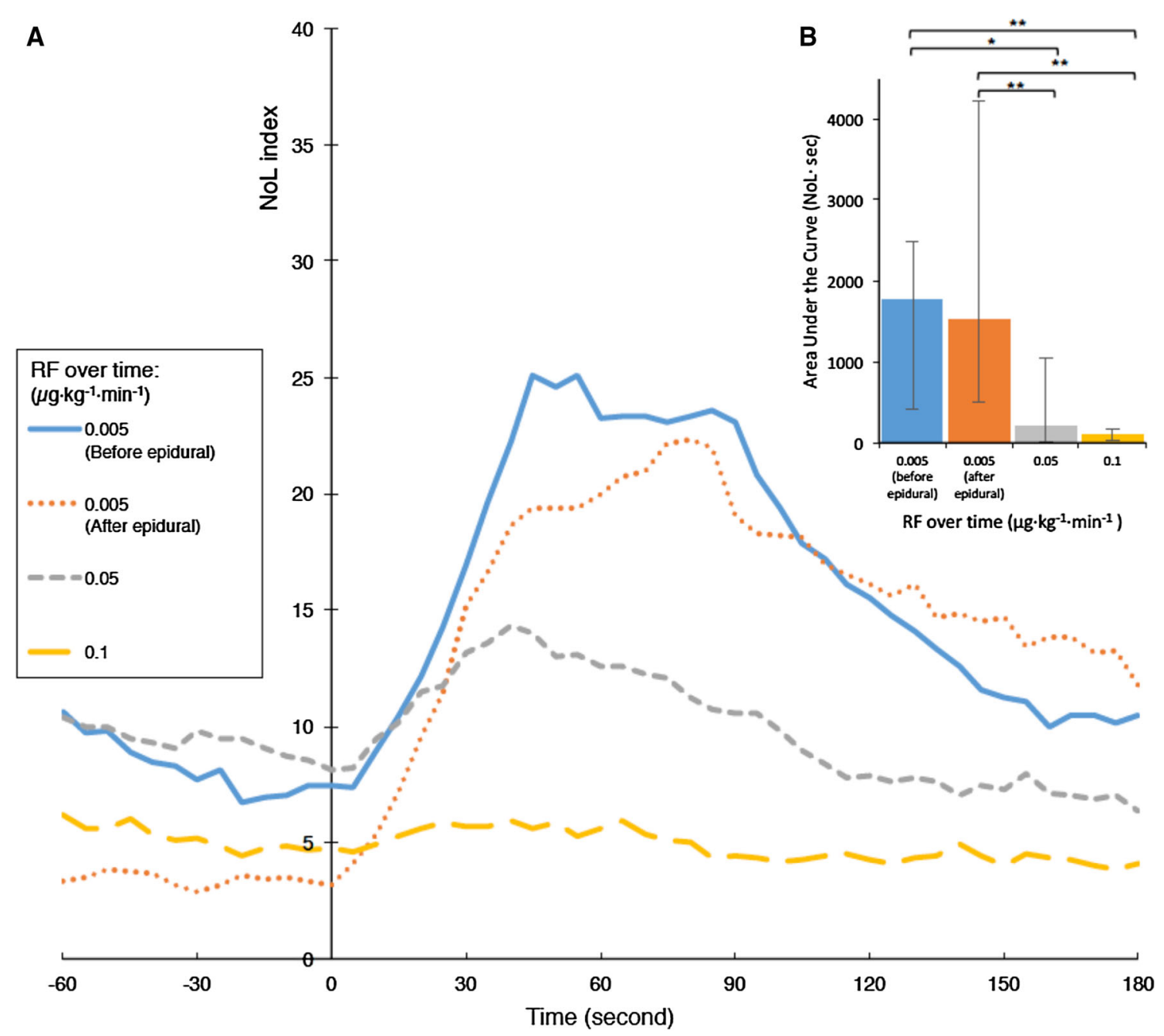

Fig. 4 Nociception Level (NOL) index trend over time before and after tetanic stimuli. A) mean NOL index value at each five seconds on a 240 -sec timeframe during experimental stimuli at different remifentanil (RF) doses. Time 0 seconds corresponds to stimulation. B) Area under the curve (AUC) of the post-stimulation period at

and BIS (AUC, 0.55 ; 95\% CI, 0.44 to 0.65 ) AUCs in our study.

Martini et al. found a NOL index AUC of 0.84, and Edry et al. found a better AUC of $0.93 .^{26,27}$ Both studies also found that NOL was better than any other studied parameters. Such comparisons suggest that, with its multiparametric approach, the NOL index may be a promising tool to accurately and reliably assess nociception. ${ }^{35}$ The question regarding the NOL cut-off value of nociceptive detection is still pending. In our study, we found maximal Youden's index values from noxious and non-noxious periods at two different electrical stimulations performed at 0.005 $\mu \mathrm{g} \cdot \mathrm{kg}^{-1} \cdot \mathrm{min}^{-1}$ (the lowest dose of intravenous remifentanil infusion) at a cut-off value of 10 . This different RF doses presenting median value with error bars corresponding to $25^{\text {th }}$ and $75^{\text {th }}$ percentiles. Friedman test significant $(P<0.0001)$. Post-hoc analysis using Dunn Bonferroni with significance at $* P<0.05, * * P<0.001$

cut-off value is lower than the threshold values of 15 and 16 found in previous studies by Ben-Israel et al. and Martini et al., respectively. ${ }^{25,27}$ Nevertheless, both studies used clinical stimuli (intubation and incision), in a protocol without low thoracic epidural placement, to build their ROC curves. Those differences could explain, in part, the difference in NOL threshold values observed. In clinical practice, under clinical stimuli, the targeted NOL index threshold to guide analgesia on NOL monitoring would probably range between 10 and 20 for best-balanced analgesia.

The study conducted by Martini et al. found a larger AUC using the variation of NOL values $(\triangle N O L$; AUC, 0.95). ${ }^{27}$ Conversely, Edry et al. showed that the absolute value of NOL was more accurate in discriminating 
Fig. 5 Nociception Level (NOL) index trend over time before and after clinical stimuli: intubation and incision. A) Incision was performed when the epidural space was already loaded with local anesthetics, explaining the small reaction of the NOL index. B) Area under the curve of NOL index for intubation and incision

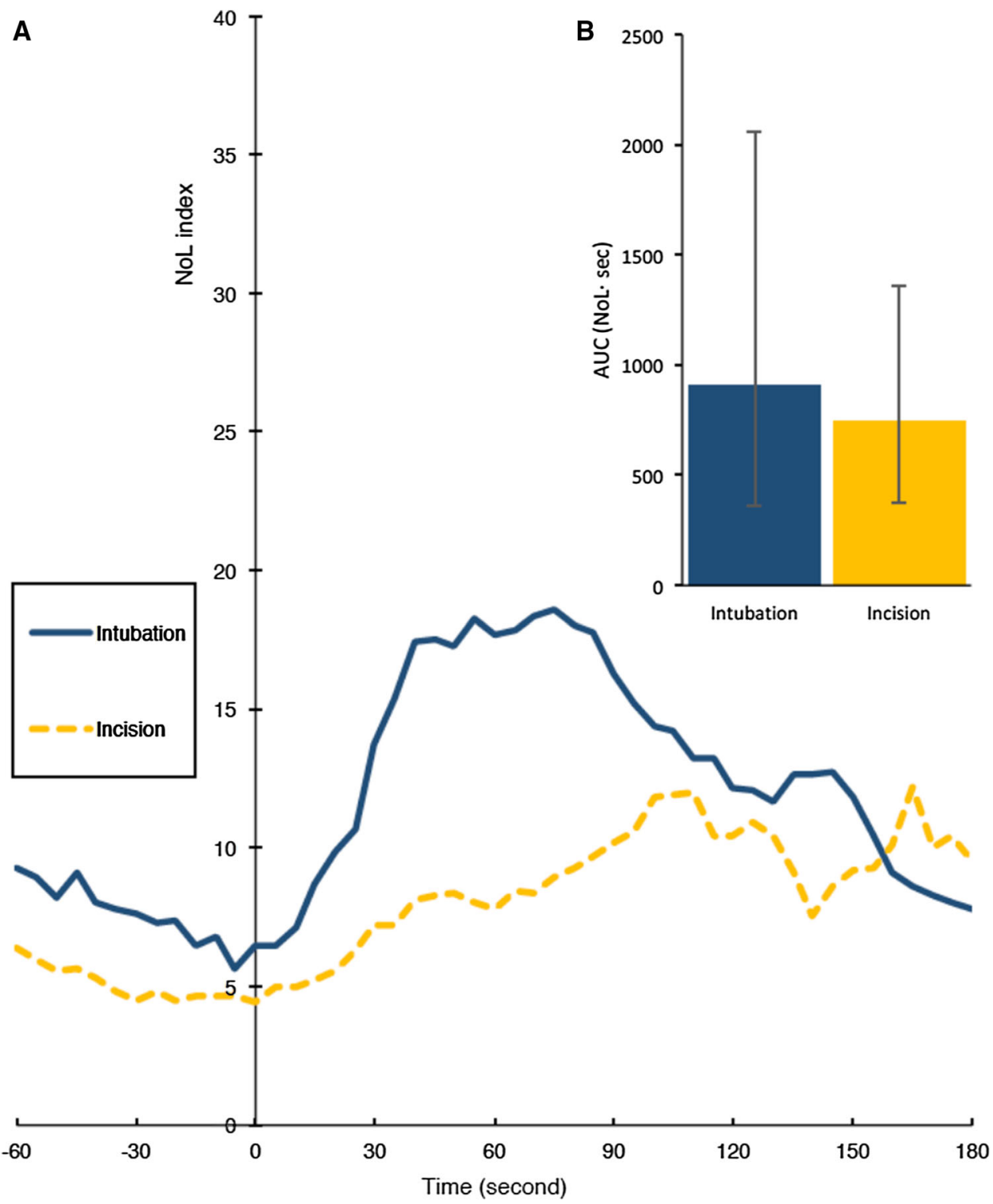

Table 3 Incidence of the highest variations of heart rate, arterial blood pressure, Bispectral Index, and Nociceptive Level Index after experimental stimuli

\begin{tabular}{lllll}
\hline & Stim1 & Stim2 & Stim3 & Stim 4 \\
\hline Remifentanil dose $\left(\mu \mathrm{g} \cdot \mathrm{kg}^{-1} \cdot \mathrm{min}^{-1}\right)$ & 0.005 & 0.005 & 0.05 & 0.1 \\
Bolused epidural & No & Yes & Yes & Yes \\
$\Delta \mathrm{HR}>20 \%, n(\%)$ & $10 / 24(42 \%)$ & $13 / 25(52 \%)$ & $1 / 25(4 \%)$ & $0 / 25(0 \%)$ \\
$\Delta$ MAP $>20 \%, n(\%)$ & $8 / 23(34 \%)$ & $9 / 24(37 \%)$ & $4 / 24(16 \%)$ & $1 / 24(4 \%)$ \\
$\Delta$ BIS $>20 \%, n(\%)$ & $9 / 24(37 \%)$ & $9 / 24(37 \%)$ & $8 / 25(32 \%)$ & $7 / 25(28 \%)$ \\
NOL $>15, n(\%)$ & $22 / 26(85 \%)$ & $19 / 26(73 \%)$ & $15 / 26(57 \%)$ & $3 / 26(11 \%)$
\end{tabular}

$\Delta \mathrm{BIS}=$ delta $\mathrm{BIS}=$ post-stimulation $\mathrm{BIS}$ variation; $\Delta \mathrm{HR}=$ delta heart rate $=$ post-stimulation heart rate variation; $\Delta \mathrm{MAP}=$ delta mean arterial blood pressure $=$ post-stimulation mean arterial pressure variation; NOL = post-stimulation Nociceptive Level Index value above 15 ; in number and $\%$ of patients. Stim1, 2, 3, 4 for experimental tetanic stimulation at the forearm level. Stim = stimulation

nociception than $\triangle \mathrm{NOL} .{ }^{26}$ We did not analyze the variation in NOL around stimulus $(\Delta \mathrm{NOL})$, as the NOL value already depends on the time derivative of its parameters.
Moreover, AUC values of NOL during the four-minute study period of a stimulation were definite (Figs $4 \mathrm{~B}$ and 5B). 


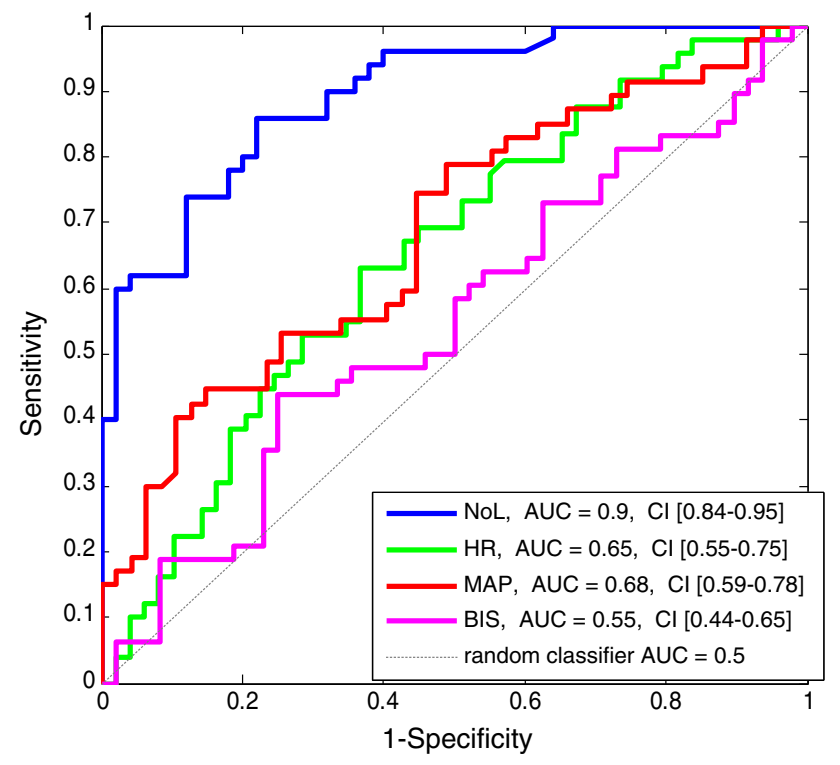

Fig. 6 Receiver operating characteristics (ROC) curve analysis at low remifentanil concentration $\left(0.005 \mu \mathrm{g} \cdot \mathrm{kg}^{-1} \mathrm{~min}^{-1}\right.$, following epidural). Discrimination of tetanic standardized electrical stimulus from non-noxious period. The maximal Youden's index value of the NOL ROC curve was calculated and reached a specificity of $88 \%$ ( $95 \%$ CI, 69.0 to 100 ) and sensitivity of $79.1 \%$ (95\% CI, 56.2 to 95.5 ). The area under the NOL ROC curve (AUC, $0.9 ; 95 \%$ CI, 0.84 to 0.95 ) was significantly different from all other variables $(P<0.0001 v s \mathrm{HR}$; $P<0.0001$ vs MAP; $P<0.0001$ vs BIS $)$. BIS $=$ bispectral index; $\mathrm{CI}=$ confidence interval; $\mathrm{HR}=$ heart rate; $\mathrm{MAP}=$ mean arterial blood pressure NOL $=$ Nociception level index

In agreement with Edry et al., we averaged poststimulation responses in a 70-sec window. ${ }^{26}$ This window, starting after the initiation of a noxious stimulus, avoids overestimating the changes in NOL values. ${ }^{14,22}$ Taking only the maximum value after stimulation might have given even better results than those shown here, but would have been much less clinically relevant.

Likelihood ratio (LR) is a simple yet recognized method to assess the accuracy of a diagnostic tool. ${ }^{36,37}$ Our results show that NOL, evaluated at a specific time-point (after epidural loading and with remifentanil infusion set at the very low dose of $0.005 \mu \mathrm{g} \mathrm{kg}^{-1} \cdot \mathrm{min}^{-1}=$ tetanic stimulation 2 of our study design, Fig. 2), had the highest positive $(\mathrm{LR}+)$ and negative (LR-) likelihood ratio for detection of a noxious stimulus compared with HR, MAP, and BIS (Table 4).

Our study has some limitations. It was powered on the primary objective, which was the correlation between NOL response and the tetanic standardized stimulus (and the doses of remifentanil infusion rate) at the time the stimulus was applied to the anesthetized patient. Accordingly, as we only studied 26 patients, it may not be enough to draw any strong conclusion about the comparison using post hoc Youden's index analysis of sensitivity and specificity between NOL, HR, and MAP (Fig. 4). Such results, even if favouring the NOL vs HR and MAP, must be considered carefully and must be confirmed by future studies that are powered on the comparison between NOL and HR and MAP for this specific sensitivity/specificity outcome.

Another statistical limitation of the study is the use of a simple Spearman correlation test to determine a positive correlation without any detail on the type of correlation (linear or not linear). A non-linear mixed effect model analysis approach to evaluate the type of correlation between NOL index response to tetanic stimuli and the doses of remifentanil infused to the patient might have been interesting. Even though we reported a statistically significant moderate correlation $(\mathrm{r}=-0.563 ; P<0.001)$, this coefficient might have even been better with another statistical approach. Also, as rank correlation fits monotonic function, it is possible to get an excellent correlation on a small segment of the function, and here, the range of measurement was small and might be one limitation of our analyses.

A further limitation was the administration of the remifentanil infusion in $\mu \mathrm{g} \mathrm{kg}^{-1} \mathrm{~min}^{-1}$ with adjusted body weight used for each patient; it might have been preferable to base remifentanil administration on the pharmacokinetic-dynamic models of remifentanil that are available in target-controlled infusion pumps, even if this is not the standard of care in North America. Another limitation was that the anesthesiologist in the OR was not blinded to the dose of remifentanil administered at each stimulation time-point and was not blinded to the NOL index reaction of these stimulations. Nevertheless, as the stimulus used (electrical tetanic stimulation) was not an operator-dependent stimulus and as all the study data were electronically recorded every five seconds, we do not believe that the operator was able to influence the poststimulation data on HR, blood pressure, BIS index, or NOL index.

Lastly, the doses of the infusion of intravenous remifentanil administered in this study were not randomized. A better design would have been to randomize these doses $(0.005,0.05$, and 0.1 $\left.\mu \mathrm{g} \cdot \mathrm{kg}^{-1} \min ^{-1}\right)$. Nevertheless, we did wait at least ten minutes at a steady infusion rate for remifentanil prior to each stimulation, therefore one dose was not likely to have a significant influence on the next one.

In conclusion, our study found that the NOL index, based on composite neurophysiologic information, showed better sensitivity and specificity to detect standardized noxious stimulus and is inversely correlated with the dose of intravenous remifentanil infusion rate. Nociception Level monitoring might be an interesting index to assess the level of nociception and monitor the efficacy of antinociception treatments in patients under GA. Other studies 
Table 4 Sensitivity, cut-off value, and likelihood ratio for the detection of experimental noxious stimulus at a specificity of $88 \%$

\begin{tabular}{lllllllll}
\hline Variable & AUC [IQR] & \multicolumn{2}{l}{ Sensitivity, \% $(95 \%$ CI $)$} & Cut-off value [IQR] & LR+ (95\% CI) & LR- (95\% CI) \\
\hline NOL & $0.90[0.84-0.95]$ & 79.1 & $(56.2$ to 95.5$)$ & $10[5.3-16.3]$ & 4.83 & $(1.69$ to 17.10$)$ & 0.52 & $(0.79$ to 0.33$)$ \\
HR & $0.65[0.55-0.75]^{*}$ & 19.1 & $(3.7$ to 43.4$)$ & $87[73.4-99.7]$ & 0 & $(0$ to 1.67$)$ & 1.13 & $(1.31$ to 0.97$)$ \\
MAP & $0.68[0.59-0.78]^{* *}$ & 35.0 & $(12.2$ to 54.3$)$ & $91[86.6-101.7]$ & 0.53 & $(0.05$ to 4.41$)$ & 1.06 & $(1.29$ to 0.85$)$ \\
BIS & $0.55[0.44-0.65]^{* * *}$ & 15.5 & $(1.9$ to 30.4$)$ & $53[49.1-60.0]$ & 0 & $(0$ to 1.91$)$ & 1.12 & $(1.31$ to 0.96$)$
\end{tabular}

Analysis at remifentanil dosage $0.005 \cdot \mathrm{kg}^{-1} \cdot \mathrm{min}^{-1}$. BIS = bispectral index; $\mathrm{CI}=$ confidence interval; $\mathrm{HR}=$ heart rate; IQR: interquartile range; $\mathrm{LR}+=$ positive likelihood ratio; $\mathrm{LR}-=$ negative likelihood ratio; $\mathrm{MAP}=$ mean arterial blood pressure; NOL $=$ Nociceptive Level index. The area under the NOL ROC curve (AUC, 0.9; 95\% CI, 0.84 to 0.95) exhibited significant (following Bonferroni correction, 3 comparison; alpha = $0.0167)$ difference compared with all other variables $(* P<0.0001 \mathrm{NOL} v s$ HR, $* * P<0.0001$ NOL $v s$ MAP, $* * * P<0.0001$ NOL $v s$ BIS)

are required to define the optimal NOL threshold values to precisely drive analgesics' administration during surgery and to evaluate the NOL ability to predict pain after surgery. Its reliability in awake patients during regional anesthesia and in intensive care units is also yet to be explored. Finally, the next major challenge will be to show an improved postoperative outcome with the intraoperative use of NOL monitoring in randomized-controlled trials.

Acknowledgements This study was supported by an Independent Investigator Initiated Trial Research Grant after a contract set by both the parties' attorneys and between the Research Center of HMR and the company Medasense LTD, Ramat Gan, Israel, and by the Department of Anesthesiology of Maisonneuve-Rosemont Hospital. Nadia Godin was the research nurse and coordinator for this study.

Conflict of interest Dr. Philippe Richebé is a member of the advisory board of the company Medasense and is a consultant for this company; as such, he perceived honorarium from 2015 until present.

Editorial responsibility This submission was handled by Dr. Hilary P. Grocott, Editor-in-Chief, Canadian Journal of Anesthesia.

Author contributions Etienne Renaud-Roy, Veronique Brulotte, Issam Tanoubi, Olivier Verdonck, and Louis-Philippe Fortier contributed to the design, execution, analysis, and revision. PierreAndre Stöckle contributed to the analysis, writing, and revision. Sarah Maximos contributed to the execution, analysis, and revision. Lucas Sideris and Pierre Dubé contributed to the design, execution, and revision. Pierre Drolet contributed to the design, analysis, writing, and revision. Rami Issa contributed to the execution and revision. Philippe Richebe is the principal investigator of the study and contributed to the design, execution, analysis, interpretation, drafting, writing, and revision of the article.

\section{References}

1. Hemmerling TM, Le N. Brief review: Neuromuscular monitoring: an update for the clinician. Can J Anesth 2007; 54: 58-72.

2. Monk TG, Weldon BC. Does depth of anesthesia monitoring improve postoperative outcomes? Curr Opin Anaesthesiol 2011; 24: $665-9$
3. Desborough JP. The stress response to trauma and surgery. Br J Anaesth 2000; 85: 109-17.

4. Ji RR, Berta T, Nedergaard M. Glia and pain: is chronic pain a gliopathy? Pain 2013; 154(Suppl 1): S10-28.

5. Latremoliere A, Woolf CJ. Central sensitization: a generator of pain hypersensitivity by central neural plasticity. J Pain 2009; 10: 895-926.

6. Angst MS, Clark JD. Opioid-induced hyperalgesia: a qualitative systematic review. Anesthesiology 2006; 104: 570-87.

7. Lee M, Silverman SM, Hansen H, Patel VB, Manchikanti L. A comprehensive review of opioid-induced hyperalgesia. Pain Physician 2011; 14: 145-61.

8. Kim SH, Stoicea N, Soghomonyan S, Bergese SD. Intraoperative use of remifentanil and opioid induced hyperalgesia/acute opioid tolerance: systematic review. Front Pharmacol 2014; 5: 108.

9. Boland JW, McWilliams K, Ahmedzai SH, Pockley AG. Effects of opioids on immunologic parameters that are relevant to antitumour immune potential in patients with cancer: a systematic literature review. Br J Cancer 2014; 111: 866-73.

10. Gach K, Wyrębska A, Fichna J, Janecka A. The role of morphine in regulation of cancer cell growth. Naunyn Schmiedebergs Arch Pharmacol 2011; 384: 221-30.

11. Inagi $T$, Suzuki $M$, Osumi $M$, Bito $H$. Remifentanil-based anaesthesia increases the incidence of postoperative surgical site infection. J Hosp Infect 2015; 89: 61-8.

12. Gruenewald $M$, Ilies $C$. Monitoring the nociception-antinociception balance. Best Pract Res Clin Anaesthesiol 2013; 27: $235-47$.

13. Logier R, Jeanne M, Tavernier B, De Jonckheere J. Pain/ analgesia evaluation using heart rate variability analysis. Conf Proc IEEE Eng Med Biol Soc 2006; 1: 4303-6.

14. Struys $M$, Vanpeteghem $C, H$, Uutela K, Blyaert N, Mortier $E P$. Changes in a surgical stress index in response to standardized pain stimuli during propofol-remifentanil infusion. Br J Anaesth 2007; 99: 359-67.

15. Storm $H$. Changes in skin conductance as a tool to monitor nociceptive stimulation and pain. Curr Opin Anaesthesiol 2008; 21: 796-804.

16. von Dincklage $F$, Correll $C$, Schneider MH, Rehberg B, Baars $J H$. Utility of nociceptive flexion reflex threshold, bispectral index, composite variability index and noxious stimulation response index as measures for nociception during general anaesthesia. Anaesthesia 2012; 67: 899-905.

17. Hamunen $K$, Kontinen $V$, Hakala E, Talke P, Paloheimo M, Kalso $E$. Effect of pain on autonomic nervous system indices derived from photoplethysmography in healthy volunteers. Br J Anaesth 2012; 108: 838-44.

18. Gruenewald M, Ilies C, Herz J, et al. Influence of nociceptive stimulation on analgesia nociception index (ANI) during 
propofol-remifentanil anaesthesia. Br J Anaesth 2013; 110: 102430.

19. Shoushtarian M, Sahinovic MM, Absalom AR, et al. Comparisons of electroencephalographically derived measures of hypnosis and antinociception in response to standardized stimuli during targetcontrolled propofol-remifentanil anesthesia. Anesth Analg 2016; 122: 382-92.

20. De Jonckheere J, Bonhomme V, Jeanne $M$, et al. Physiological signal processing for individualized anti-nociception management during general anesthesia: a review. Yearb Med Inform 2015; 10: 95-101.

21. Seitsonen E, Korhonen IK, Van Gils MJ, et al. EEG spectral entropy, heart rate, photoplethysmography and motor responses to skin incision during sevoflurane anaesthesia. Acta Anaesthesiol Scand 2005; 49: 284-92.

22. Gruenewald $M$, Meybohm $P$, Ilies $C$, et al. Influence of different remifentanil concentrations on the performance of the surgical stress index to detect a standardized painful stimulus during sevoflurane anaesthesia. Br J Anaesth 2009; 103: 586-93.

23. Treister $R$, Kliger $M$, Zuckerman $G$, Aryeh I, Eisenberg E. Differentiating between heat pain intensities: the combined effect of multiple autonomic parameters. Pain 2012; 153: 1807-14.

24. Rantanen $M$, Yli-Hankala A, van Gils $M$, et al. Novel multiparameter approach for measurement of nociception at skin incision during general anaesthesia. Br J Anaesth 2006; 96 : 367-76.

25. Ben-Israel N, Kliger $M$, Zuckerman $G$, Katz $Y$, Edry $R$. Monitoring the nociception level: a multi-parameter approach. J Clin Monit Comput 2013; 27: 659-68.

26. Edry R, Recea V, Dikust Y, Sessler DI. Preliminary intraoperative validation of the nociception level index: a noninvasive nociception monitor. Anesthesiology 2016; 125: 193-203.

27. Martini CH, Boon M, Broens SJ, et al. Ability of the nociception level, a multiparameter composite of autonomic signals, to detect noxious stimuli during propofol-remifentanil anesthesia. J Am Soc Anesthesiol 2015; 123: 524-34.
28. Julien M, Décary E, Issa R, Verdonck $O$, Fortier LP, Richebé P. Nociception Level (NoL) index alteration after standardized nociceptive stimulus decreases with higher doses of remifentanil. European Society of Anesthesiology 2016. Available from URL: https://www.medasense.com/wp-content/uploads/2017/01/ Medasense-philippe-richebe-Abstract-ESA-2016.pdf (accessed February 2019).

29. Schwartz SN, Pazin GJ, Lyon JA, Ho M, Pasculle AW. A controlled investigation of the pharmacokinetics of gentamicin and tobramycin in obese subjects. J Infect Dis 1978; 138: 499505.

30. Robinson Jd, Lupkiewicz SM, Palenik LM, Lopez L, Ariet M. Determination of ideal body weight for drug dosage calculations. Am J Health Sys Pharm 1983; 40: 1016-9.

31. Bauer LA. Applied Clinical Pharmacokinetics - Second Edition. McGraw-Hill Medical; 2008.

32. Rantanen M, Yppärilä-Wolters $H$, van Gils $M$, et al. Tetanic stimulus of ulnar nerve as a predictor of heart rate response to skin incision in propofol-remifentanil anaesthesia. Br J Anaesth 2007; 99: 509-13.

33. Cohen J. Statistical Power Analysis for the Behavioral Sciences. Hillsdale, NJ: Lawrence Earlbaum Associates; 1988. p. 20-6.

34. Nakagawa S, Cuthill IC. Effect size, confidence interval and statistical significance: a practical guide for biologists. Biol Rev Camb Philos Soc 2007; 82: 591-605.

35. Cowen R, Stasiowska MK, Laycock H, Bantel C. Assessing pain objectively: the use of physiological markers. Anaesthesia 2015; 70: $828-47$

36. Altman DG, Bland JM. Diagnostic tests 2: predictive values. BMJ 1994; 309: 102.

37. McGee S. Simplifying likelihood ratios. J Gen Intern Med 2002; 17: $647-50$.

Publisher's Note Springer Nature remains neutral with regard to jurisdictional claims in published maps and institutional affiliations. 